ANNALES

POLONICI MATHEMATICI

$89.2(2006)$

\title{
A domain whose envelope of holomorphy is not a domain
}

\author{
by Edgar Lee Stout (Seattle, WA)
}

\begin{abstract}
We construct a domain of holomorphy in $\mathbb{C}^{N}, N \geq 2$, whose envelope of holomorphy is not diffeomorphic to a domain in $\mathbb{C}^{N}$.
\end{abstract}

The envelope of holomorphy plays a central role in multivariate complex analysis; the standard textbooks in several complex variables give various constructions for it. Given a domain $D$ in $\mathbb{C}^{N}$, the envelope of holomorphy of $D$ is a Riemann domain $\left(D^{*}, \pi\right)$ so that $\pi: D^{*} \rightarrow \mathbb{C}^{N}$ is a locally biholomorphic map. Roughly speaking, $\left(D^{*}, \pi\right)$ is the largest Riemann domain to which all functions holomorphic on $D$ extend. The manifold $D^{*}$ is presented as an abstractly given complex manifold, and, in general, the map $\pi$ is not injective. Thus, the manifold $D^{*}$ is not presented as a domain in $\mathbb{C}^{N}$. The question arose in a recent discussion with some colleagues whether $D^{*}$ might, nonetheless, be biholomorphically equivalent to a domain in $\mathbb{C}^{N}$.

It is the purpose of this note to exhibit a domain $\Omega$ in $\mathbb{C}^{N}, N \geq 2$, whose envelope of holomorphy is not biholomorphically equivalent to a domain in $\mathbb{C}^{N}$. The construction of $\Omega$ requires the following lemma, in which $\mathbb{B}_{n}$ denotes the open unit ball in $\mathbb{C}^{n}$ centered at the origin.

Lemma. In $\mathbb{C}^{n}, n \geq 2$, let

$$
D_{\varepsilon}=\left\{z=x+i y \in \mathbb{C}^{n}=\mathbb{R}_{x}^{n}+i \mathbb{R}_{y}^{n}: z \in \mathbb{B}_{n} \text { and }|y|>\varepsilon\right\} .
$$

If $K$ is a compact subset of $\mathbb{B}_{n}$, then for sufficiently small $\varepsilon>0$ there is a domain $\widetilde{D}_{\varepsilon} \subset \mathbb{B}_{n}$ that contains $D_{\varepsilon}$ and $K$, and with the property that each function holomorphic on $D_{\varepsilon}$ continues holomorphically into $\widetilde{D}_{\varepsilon}$.

Precisely: The fixed domain $\widetilde{D}_{\varepsilon}$ has the property that for each function $f$ holomorphic on $D_{\varepsilon}$ there is a corresponding (single-valued) function $\widetilde{f}$ holomorphic on $\widetilde{D}_{\varepsilon}$ that is an extension of $f$.

2000 Mathematics Subject Classification: Primary 32D10.

Key words and phrases: envelope of holomorphy. 
Proof. The set $b \mathbb{B}_{n} \cap\{z: y=0\}$ is the $(n-1)$-sphere $\mathbb{S}^{n-1}=b \mathbb{B}_{n} \cap \mathbb{R}_{x}^{n}$, which, as a compact subset of $\mathbb{R}^{n}$, is polynomially convex. Consequently, there is $\varepsilon>0$ small enough that the polynomially convex hull of the set $S_{\varepsilon}=b \mathbb{B}_{n} \cap\{z:|y| \leq \varepsilon\}$ is contained in $\overline{\mathbb{B}}_{n} \backslash K$. Thus, if $\varepsilon>0$ is small, then $\widehat{S}_{\varepsilon}$, the polynomially convex hull of the set $S_{\varepsilon}$, is disjoint from $K$. A function holomorphic on $D_{\varepsilon}$ continues holomorphically into $\mathbb{B}_{n} \backslash \widehat{S}_{\varepsilon}$. For this continuation, one can consult the appendix to [5] or [1]. For the $\widetilde{D}_{\varepsilon}$ of the lemma, we can take the latter set.

We now proceed to the construction of $\Omega$.

Fix an integer $N \geq 2$. The space $\mathbb{C}^{N}$ contains $N$-dimensional compact totally real submanifolds, e.g., the unit torus $\mathbb{T}^{N}$, which is the distinguished boundary of the unit polydisc in $\mathbb{C}^{N}$. The paper [6] - see in particular Lemmas 3,4, and 5-therefore provides in $\mathbb{C}^{N}$ a pair $M_{1}$ and $M_{2}$ of compact, connected, totally real $N$-dimensional submanifolds of class $\mathscr{C}^{\infty}$ with the following properties:

(a) $M_{1} \cap M_{2}$ consists of two points, say $p_{1}$ and $p_{2}$.

(b) In a neighborhood of $p_{1}$ and in a neighborhood of $p_{2}$ the manifolds $M_{1}$ and $M_{2}$ coincide with their tangent planes and these tangent planes are transversal.

(c) For small balls $B_{j}$ centered at $p_{j}$, each of the intersections $\bar{B}_{j} \cap$ $\left(M_{1} \cup M_{2}\right)$ is polynomially convex.

It is further shown that

(d) The union $M_{1} \cup M_{2}$ has a neighborhood basis consisting of Stein domains.

Let us denote the union $M_{1} \cup M_{2}$ by $\Sigma$. That $\Sigma$ has a Stein neighborhood basis implies that it is holomorphically convex in the sense of Harvey and Wells [2], i.e., that every nonzero complex homomorphism of the algebra $\mathscr{O}(\Sigma)$ of germs of functions holomorphic on $\Sigma$ is of the form $f \mapsto f(p)$ for some necessarily unique point $p \in \Sigma$. Alternatively phrased, $\Sigma$ is, in a natural way, the spectrum of the algebra $\mathscr{O}(\Sigma)$. As a consequence of the holomorphic convexity of $\Sigma$, we can invoke an approximation theorem of O'Farrell, Preskenis, and Walsh [4, Theorem 2] to conclude that every continuous function on $\Sigma$ can be approximated uniformly on $\Sigma$ by functions holomorphic on varying neighborhoods of $\Sigma$.

Without loss of generality, we can suppose that the point $p_{1}$ from property (a) above is the origin. We can then choose coordinates so that the tangent space $T_{0} M_{1}$ is $\mathbb{R}^{N}$ and so that in the unit ball $\mathbb{B}_{N}$ centered at 0 , the manifolds $M_{1}$ and $M_{2}$ both coincide with their tangent planes, which means that $M_{1} \cap \overline{\mathbb{B}}_{N}=\mathbb{R}^{N} \cap \overline{\mathbb{B}}_{N}$ and that $M_{2} \cap \overline{\mathbb{B}}_{N}=T_{0} M_{2} \cap \overline{\mathbb{B}}_{N}$. 
Let $\gamma: \mathbb{T} \rightarrow \Sigma$ map the unit circle $\mathbb{T}$ in the plane into $\Sigma$ homeomorphically and in such a way that $\gamma(1)=0, \gamma(-1)=p_{2}$, and so that $\gamma$ carries the upper half of $\mathbb{T}$ into $M_{1}$, and the lower half into $M_{2}$. The map $\gamma$ is not homotopically trivial in $\Sigma$.

We construct a continuous function $\varphi: \Sigma \rightarrow \mathbb{T}$ with $\varphi \circ \gamma\left(e^{i t}\right)=e^{i t}$ for all points $e^{i t} \in \mathbb{T}$. There is such a function: By replacing $\varphi$ by $\varphi /|\varphi|$ if necessary, we see that it suffices to find a zero-free function $\varphi$ on $\Sigma$ such that $\varphi \circ \gamma\left(e^{i t}\right)=e^{i t}$ for all points $e^{i t} \in \mathbb{T}$. To do this, let $\lambda_{1}$ and $\lambda_{2}$ be continuous real-valued functions on $M_{1}$ and $M_{2}$, respectively, that satisfy $\lambda_{1} \circ \gamma\left(e^{i t}\right)=t$ when $0 \leq t \leq \pi$ and $\lambda_{2} \circ \gamma\left(e^{i t}\right)=t$ when $\pi \leq t \leq 2 \pi$. The function $\varphi$ that agrees on $M_{1}$ with $e^{i \lambda_{1}}$ and on $M_{2}$ with $e^{i \lambda_{2}}$ is continuous and zero-free on $\Sigma$ and satisfies $\varphi \circ \gamma\left(e^{i t}\right)=e^{i t}$ for all $t$. By construction, $\varphi \mid M_{1}$ is homotopic to a constant as is $\varphi \mid M_{2}$. (That $\varphi \mid M_{1}$ is homotopic to a constant, is immediate: The map $H:[0,1] \times M_{1} \rightarrow \mathbb{T}$ given by $H(t, x)=e^{i t \lambda_{1}(x)}$ is a homotopy connecting $H(1, \cdot)=\varphi=e^{i \lambda_{1}}$ to the constant map $H(0, \cdot)$.)

By the approximation theorem of O'Farrell, Preskenis, and Walsh, the function $\varphi$ can be approximated uniformly on $\Sigma$ by functions holomorphic on varying neighborhoods of $\Sigma$. There is, therefore, a neighborhood $\Omega_{0}$ of $\Sigma$ on which there is a zero-free holomorphic function $f_{0}$ such that the map $f_{0} \circ \gamma: \mathbb{T} \rightarrow \mathbb{C} \backslash\{0\}$ is homotopic to the inclusion $\mathbb{T} \hookrightarrow \mathbb{C} \backslash\{0\}$, such that $\Omega_{0}=\Omega_{0,1} \cup \Omega_{0,2}$ with $\Omega_{0,1}$ and $\Omega_{0,2}$ domains that contain $M_{1}$ and $M_{2}$, respectively, such that $f_{0} \mid \Omega_{0,1}$ and $f_{0} \mid \Omega_{0,2}$ have holomorphic logarithms, say $\ell_{1}$ and $\ell_{2}$. These logarithms can be chosen so that $\ell_{1}\left(p_{2}\right)=\ell_{2}\left(p_{2}\right)$. With this choice, $\ell_{1}(0)=\ell_{2}(0) \pm 2 \pi i$.

Let $\Omega \subset \Omega_{0}$ be a domain of the form $W_{1} \cup W_{2}$, where $W_{1}$ is a thin ribbon around $M_{1}$ that is contained in $\Omega_{0,1}$ and that satisfies $W_{1} \cap \mathbb{B}_{N} \subset$ $\left\{z=x+i y \in \mathbb{R}_{x}^{N}+i \mathbb{R}_{y}^{N}:|y|<\eta\right\}$ for a small $\eta>0$ whose size will be specified further below. The domain $W_{2}$ is constructed in the following way. The domain $\Omega_{0,2}$ contains a ball $\mathbb{B}(0, r)$ of some radius $r>0$ centered at the origin. We take $r$ to be less than 1 so that at points of $\mathbb{B}_{N}(0, r)$ the manifold $M_{2}$ agrees with its tangent space. Having fixed $r$, we introduce the set

$$
\Delta_{r, \varepsilon}=\left\{z=x+i y \in\left(\mathbb{R}_{x}^{N}+i \mathbb{R}_{y}^{N}\right) \cap \mathbb{B}_{N}(0, r): y>|\varepsilon|\right\} .
$$

We insist that our $\varepsilon$ be so small that the tangent space $T_{0} M_{2}$ meets the sphere $b \mathbb{B}_{N}(0, r)$ in a set that is contained in $\bar{\Delta}_{r, \varepsilon}$. The Lemma proved above shows that there is a domain $D_{0}$ containing $\Delta_{r, \varepsilon}$ and $\mathbb{B}_{N}(0, r) \cap T_{0} M_{2}$ such that every function $f$ that is holomorphic on $\Delta_{r, \varepsilon}$ continues holomorphically into $D_{0}$.

Our domain $W_{2}$ is defined by

$$
W_{2}=\left(V \backslash \mathbb{B}_{N}(0, r)\right) \cup \Delta_{r, \varepsilon},
$$

in which $V \subset \Omega_{0}$ is a thin tube around the manifold $M_{2}$ that is contained in $\Omega_{0,2}$. 
We now choose the $\eta$ used in the definition of $W_{1}$ to be smaller than the $\varepsilon$ used in the definition of $W_{2}$. This has the effect that $W_{1}$ and $\Delta_{r, \varepsilon}$ are disjoint and, indeed, have disjoint closures.

As noted already, our domain $\Omega$ is the union $W_{1} \cup W_{2}$.

The envelope of holomorphy of $\Omega$ is a Riemann domain $\left(\Omega^{*}, \pi\right)$. It has the property that $\pi\left(\Omega^{*}\right) \supset \Omega$, but more than that, $\pi\left(\Omega^{*}\right)$ also contains the domain $D_{0}$.

Let $\iota: \Omega \rightarrow \Omega^{*}$ be the canonical injection, so that $\pi \circ \iota$ is the identity map on $\Omega$, and for every $f$ holomorphic on $\Omega$, there is a unique $f^{*}$ holomorphic on $\Omega^{*}$ that satisfies $f=f^{*} \circ \iota$.

The map $\iota$ carries the manifold $M_{1}$ onto a submanifold $M_{1}^{*}$ of $\Omega^{*}$. It also carries the open subset $M_{2,+}=M_{2} \backslash\left(\mathbb{B}_{N}(0, r) \cap\{z:|y| \leq \varepsilon\}\right)$ onto a locally closed $\left({ }^{1}\right)$ submanifold $M_{2,+}^{*}$ of $\Omega^{*}$ that meets $M_{1}^{*}$ at a single point, viz., at the point $\iota\left(p_{2}\right)$, and the intersection there is transversal.

Because the manifold $\Omega^{*}$ is a Stein manifold, there is a map $j: D_{0} \rightarrow \Omega^{*}$ that agrees on $\Delta_{r, \varepsilon}$ with $\iota$.

The existence of $j$ is seen as follows. Since $\Omega^{*}$ is a Stein manifold, we can assume it to be a complex submanifold of $\mathbb{C}^{m}$ for a sufficiently large $m$. The map $\iota$ is defined on $\Delta_{r, \varepsilon}$, and, with $\Omega^{*} \subset \mathbb{C}^{m}$, it is given by an $m$-tuple $\left(\iota_{1}, \ldots, \iota_{m}\right)$ of holomorphic functions. Each $\iota_{s}$ extends to a holomorphic function $j_{s}$ on $D_{0}$. The map $j$ is then the $m$-tuple $\left(j_{1}, \ldots, j_{m}\right)$.

It carries the totally real disc $\Gamma=T_{0} M_{2} \cap \mathbb{B}_{N}(0, r)$ onto a locally closed submanifold $\Gamma^{*}$ of $\Omega^{*}$. The set $\Gamma^{*} \cup M_{2,+}^{*}$ is a smooth submanifold - call it $M_{2}^{*}$ - of $\Omega^{*}$ that is diffeomorphic to the manifold $M_{2}$.

The manifolds $M_{1}^{*}$ and $M_{2}^{*}$ meet only at the point $\iota\left(p_{2}\right)$, which we denote by $p_{2}^{*}$. Because the map $\iota$ is injective, the intersection $M_{1}^{*} \cap M_{2}^{*}$ is necessarily contained in the set $\pi^{-1}\left(M_{1} \cap M_{2}\right)=\pi^{-1}(0) \cup\left\{p_{2}^{*}\right\}$. The point now is that $\iota(0)$ is different from $j(0)$ as follows from the existence of the function $f_{0}$ that we constructed above. To prove this, we define a function $\psi$ by the condition that $\psi=\ell_{1}$ on $W_{1}$ and $\psi=\ell_{2}$ on $W_{2}$. This function is well defined and holomorphic on $\Omega$, and it is a branch of $\log f_{0}$ on $\Omega$. Denote by $\psi_{0}$ the continuation of $\psi \mid \Delta_{r, \varepsilon}$ into the domain $D_{0}$. The value of $\psi_{0}$ at 0 differs by $\pm 2 \pi i$ from the value of $\psi$ at 0 . This means that the points $\iota(0)$ and $j(0)$ differ. Therefore the manifolds $M_{1}^{*}$ and $M_{2}^{*}$ meet at a single point in $\Omega^{*}$.

Intersection theory [3, middle of p. 132] shows that in $\mathbb{C}^{N}$ it is impossible for two $N$-dimensional compact smooth manifolds to intersect at a single point if the intersection is transversal, so the domain $\Omega^{*}$ is not biholomorphically equivalent to a domain in $\mathbb{C}^{N}$.

This completes our discussion of the announced domain $\Omega$.

$\left({ }^{1}\right)$ A set is locally closed if it is a closed subset of an open subset of the ambient space. 
This discussion gives rise to an obvious and probably difficult question: Can one give conditions under which the envelope of holomorphy of a domain in $\mathbb{C}^{N}$ is biholomorphically equivalent to a domain in $\mathbb{C}^{N}$ ? Note that this is not the question of when the envelope of holomorphy is schlicht. Precisely, the latter question, which is classical and not easy, is this: If $D$ is a domain in $\mathbb{C}^{N}$ with envelope of holomorphy $(\widetilde{D}, \pi)$, what conditions on $D$ guarantee that the projection $\pi$ is injective?

\section{References}

[1] E. M. Chirka and E. L. Stout, Removable singularities in the boundary, in: Contributions to Complex Analysis and Analytic Geometry, Vieweg, Braunschweig, 1994, 43-104.

[2] F. R. Harvey and R. O. Wells Jr., Compact holomorphically convex subsets of a Stein manifold, Trans. Amer. Math. Soc. 136 (1969), 509-516.

[3] M. W. Hirsch, Differential Topology, Springer, New York, 1976.

[4] A. G. O'Farrell, K. J. Preskenis and D. Walsh, Holomorphic approximation in Lipschitz norms, in: Proc. Conf. on Banach Algebras and Several Complex Variables (New Haven, CT, 1983), Contemp. Math. 32, Amer. Math. Soc., Providence, RI, 1984, 187-194.

[5] J.-P. Rosay and E. L. Stout, Radó's theorem for CR-functions, Proc. Amer. Math. Soc. 106 (1989), 1017-1026.

[6] E. L. Stout and W. R. Zame, Totally real imbeddings and the universal covering spaces of domains of holomorphy: some examples, Manuscripta Math. 50 (1985), 29-48.

The University of Washington

Seattle, WA 98195, U.S.A.

E-mail: stout@math.washington.edu

Received 9.5.2006

and in final form 3.7.2006 